\title{
The calibration and uncertainty evaluation by elementary measurement models
}

\author{
Samoilenko Oleksandr ${ }^{1}$ \\ ${ }^{1} \mathrm{SE} \ll$ Ukrmetrteststandard», Ukraine \\ E-mail: asam@ukrcsm.kiev.ua
}

\begin{abstract}
Nature has often made necessary things simple (elementary) and complicated ones unnecessary. This can be applied to measurement models of the large amount of working measurement standards and working measuring instruments. Nevertheless, if measurement model is elementary, it does not mean that it is primitive. It should be formulated according to the sufficiency, mathematical completeness and correctness principles. The attempt to formulate models according to the mentioned principles is introduced.

Models are called elementary, as measurement result is function of one or two homogeneous measured quantities. Thus, measurement result is a single reading of the measurement standard or measuring instrument or average value of several readings, or bias as the disparity between readings of the calibrated measuring instrument and measurement standard.

Notwithstanding the elementary measurement models simplicity, many variants of solutions are obtained in the process of the measurement result uncertainty evaluation by these models. Publication demonstrates how to choose the best uncertainty evaluation from many variants of solutions depending on whether single readings or average of several readings is included to measurement model. The best choice of the measurement model depends on resolution of the indicating measuring instrument. Moreover, the best choice depends on the measurement standard used for calibration, which is measure or measuring instrument and depends on the calibration object: measure or measuring instrument.
\end{abstract}

Keywords: calibration, elementary measurement models, uncertainty, working measurement standard, working measuring instrument.

\section{Foreword}

Discussions concerning the necessity of standardization of the great amount of calibration instructions sometimes arise among the metrologists. For some instruments used for the intended purpose in certain areas, this is useful. Though, in general this imposes certain restrictions, that contradicts to the philosophy of the calibration in terms of its use flexibility. Presented in publication recommendations should be considered as the author's point of view on the philosophy of the calibration flexibility, but not as something stable and invariable. Presented measurement models may be used as they are, but they should be also considered as the aid for development of the own models in particular cases of calibration and operation according to the circumstances.

\section{Introduction}

This publication does not cover the variety of cases concerning choice of measurement models as well as processing methods of their results including measurement uncertainty evaluation. Thus, they may be used for calibration methods development of numerous kinds of measuring instruments, which are 
working measurement standards (measuring instruments and measures) and working measuring instruments.

First of all, let us define more precisely terms used in this publication. Thus, calibrated instrument or measure becomes measuring standard - instrument or measuring standard - measure after calibration. If instrument is not assigned to calibration of other instruments, then it is assigned to use in operation. Use of the calibrated instrument for instruments of lower rank calibration is also operation. Though, term instrument in this publication means the instrument used in operation, measurement or testing laboratory. Comparators are also instruments used for measures calibration, but they are called only comparators.

The aim of this publication is the analysis of measurement uncertainty components by elementary measurement models in the process of working measurement standards and working measuring instruments calibration.

This allows obtaining the best uncertainty evaluations from many variants, which are not overestimated or underestimated.

Subsection 4.1.1 [1] begins with the words «In most cases, a measurand $Y$ is not measured directly...». Nevertheless, great number of working measurement standards and working measuring instruments is used for single direct measurements. This article is dedicated to those working measurement standards and measuring instruments.

Note to subsection 4.1.2 [1] «Equation (1) may be as elementary as $Y=X_{1}-X_{2}$. This expression models, for example, the comparison of two determinations of the same quantity $X »$. Actually, this elementary direct measurement model can be used in the process of many working measurement standards or working measuring instruments calibration.

Elementary models have one or two homogenous evaluated parameters in the measurement point. They do not need formulating and solving the system equations. Parameter for elementary models is as a rule the measuring instrument reading, average or weighted average value of the measuring instrument readings or bias in the measurement point as the difference between the direct measurement by measuring instrument and measurement standard.

Many variants for the uncertainty of measurement result evaluation by the elementary measurement models arise. Choice of the particular solution depends on whether the single readings or average of several readings is included to measurement model. It also depends on the measurement standard used for calibration, which is measure or measuring instrument and depends on the calibration object: measure or measuring instrument. Choice of the solution also depends on resolution of the indicating measuring instrument.
3. Elementary measurement model in the process of working measurement standard or measuring instrument calibration

In the process of measurement standard or measuring instrument calibration by elementary measurement model, biases of measurement standard or measuring instrument readings and their uncertainties are estimated in discrete points. These bias quantities with the opposite sign are used as corrections in the process of other instruments calibration or for routine measurements during the operation process.

Elementary measurement model for final calibration result is:

$$
y_{i}=d_{i} \text { or } y_{i}=-\Delta x_{i}
$$

where

$y_{i}$ is the measurement result;

$d_{i}$ is the bias of measuring instrument readings in the point number $i$;

$\Delta x_{i}=-d_{i}$ is correction to the measuring instrument readings $x_{i}$ in the same point number $i$, in which calibration was provided.

Elementary measurement model (1) means that bias is accepted as measurement result and bias uncertainty is accepted as measurement uncertainty.

Elementary measurement model (1) may be expressed by one of the formulae:

$$
d_{i}=x_{i l}-x_{i l}^{e t}
$$

or

$$
d_{i}=\bar{x}_{i}-x_{i l}^{e t}
$$

or

$$
d_{i}=x_{i 1}-\bar{x}_{i}^{e t}
$$

or

$$
d_{i}=\bar{x}_{i}-\bar{x}_{i}^{e t}
$$

where

$x_{i 1}^{e t}$ is single (the first and the last one) reading of the measuring standard in the point number $i$ of the measurement range or quantity value assigned to measure according to its calibration; $x_{i 1}$ is single (the first and the last one) reading of the calibrated measuring instrument in the point number $i$ of the measurement range or quantity value that should be assigned to measure according its calibration;

$\bar{x}_{i}^{e t}$ is the average of measurement standard readings in the point number $i$ of the measurement range;

$\bar{x}_{i}$ is the average of the calibrated measuring instrument readings in the point number $i$ of the measurement range. 
Average values of several readings of the measurement standard and measuring instrument (calibrated instrument) are calculated by the known formulae:

$$
\bar{x}_{i}=\frac{\sum_{l=1}^{m} x_{i l}}{m} ; \bar{x}_{i}^{e t}=\frac{\sum_{l=1}^{m} x_{i l}^{e t}}{m},
$$

where

$x_{i l}$ is measuring instrument reading number $l$ in the point number $i$ of the measurement range;

$x_{i l}^{e t}$ is measurement standard reading number $l$ in the point number $i$ of the measurement range; $m$ is the quantity of measurements provided in the point number $i$ of the measurement range;

$l=1, \ldots, l, \ldots, m$ is sequence number of the measurement.

Biases with the opposite sign should be used as corrections from the calibration certificate to measurement standard readings. Measurement standard and measuring instrument readings corrections should be specified by calibration instruction and/or manual, etc. For example, corrections of environment influence on measurement standard and/or measuring instrument. Their uncertainties are included to the uncertainty budget, but they purposely are not considered below.

Experimental standard deviationi of measurement standard and measuring instrument single reading in particular measurement point:

$$
\begin{gathered}
u_{A}\left(x_{i l}\right)=S\left(x_{i l}\right)=\sqrt{\frac{\sum_{l=1}^{m}\left(x_{i l}-\bar{x}_{i}\right)^{2}}{m-1} ;} \\
u_{A}\left(x_{i l}^{e t}\right)=S\left(x_{i l}^{e t}\right)=\sqrt{\frac{\sum_{l=1}^{m}\left(x_{i l}^{e t}-\bar{x}_{i}^{e t}\right)^{2}}{m-1}} .
\end{gathered}
$$

Experimental standard deviations $u_{A}\left(x_{i l}\right)$ and $u_{A}\left(x_{i l}^{e t}\right)$ are accepted as type A standard uncertainties $S\left(x_{i l}\right)$ and $S\left(x_{i l}^{e t}\right)$ of measurement standard and measuring instrument single reading.

Experimental standard deviations of measurement standard and measuring instrument readings average in particular measurement point:

$$
\begin{aligned}
u_{A}\left(\bar{x}_{i}\right)= & S\left(\bar{x}_{i}\right)=\sqrt{\frac{\sum_{l=1}^{m}\left(x_{i l}-\bar{x}_{i}\right)^{2}}{m \cdot(m-1)}}= \\
& =\frac{u_{A}\left(x_{i l}\right)}{\sqrt{m}}=\frac{S\left(x_{i l}\right)}{\sqrt{m}} ;
\end{aligned}
$$

$$
\begin{aligned}
u_{A}\left(\bar{x}_{i}^{e t}\right) & =S\left(\bar{x}_{i}^{e t}\right)=\sqrt{\frac{\sum_{l=1}^{m}\left(x_{i l}^{e t}-\bar{x}_{i}^{e t}\right)^{2}}{m \cdot(m-1)}}= \\
& =\frac{u_{A}\left(x_{i l}^{e t}\right)}{\sqrt{m}}=\frac{S\left(x_{i l}^{e t}\right)}{\sqrt{m}} .
\end{aligned}
$$

Experimental standard deviations $S\left(\bar{x}_{i}\right)$ and $S\left(\bar{x}_{i}^{e t}\right)$ are accepted as type A standard uncertainties $u_{A}\left(\bar{x}_{i}\right)$ and $u_{A}\left(\bar{x}_{i}^{e t}\right)$ of measurement standard and measuring instrument readings average.

If measurement model is defined by formula (5) and readings are taken synchronously, then calculating standard deviation of the pairs of readings it is necessary to evaluate their correlation. For example, the pairs of readings correlation exist in the process of thermometer calibration in thermostat, when readings of measurement standard thermometer and the calibrated one are taken simultaneously due to the instability of thermostat environment. Uncertainty of such measurements should be evaluated including correlation between the measurement standard and calibrated measuring instrument readings. In complicated cases, formulae of section 5.1 [1] or Annex D [2] or formulae from other sources should be used. For elementary models, covariance moment $K\left(x_{i l}^{e t}, x_{i l}\right)$ and correlation coefficient $r$ should be calculated to this purpose:

$$
\begin{gathered}
K\left(x_{i l}^{e t}, x_{i l}\right)=\frac{\sum_{l=1}^{m}\left(x_{i l}^{e t}-\bar{x}_{i}^{e t}\right) \cdot\left(x_{i l}-\bar{x}_{i}\right)}{m-1} ; \\
r=\frac{K\left(x_{i l}^{e t}, x_{i l}\right)}{S\left(x_{i l}^{e t}\right) \cdot S\left(x_{i l}\right)} .
\end{gathered}
$$

Experimental standard deviation (type A standard uncertainty) of single bias $d_{i l}=x_{i l}-x_{i l}^{e t}$ in particular measurement point:

$$
\begin{gathered}
u_{A}\left(d_{i l}\right)=S\left(x_{i l}^{e t}, x_{i l}\right)= \\
=\sqrt{u_{A}\left(x_{i l}^{e t}\right)^{2}+u_{A}\left(x_{i l}\right)^{2}-2 \cdot r \cdot u_{A}\left(x_{i l}^{e t}\right) \cdot u_{A}\left(x_{i l}\right)} .
\end{gathered}
$$

Experimental standard deviation (type A standard uncertainty) of average bias $d_{i}$ in particular measurement point:

$$
u_{A}\left(d_{i}\right)=S\left(\bar{x}_{i}^{e t}, \bar{x}_{i}\right)=\frac{u_{A}\left(x_{i l}^{e t}, x_{i l}\right)}{\sqrt{m}}=\frac{S\left(x_{i l}^{e t}, x_{i l}\right)}{\sqrt{m}} .
$$

Readings correlation should be not ignored. Let us imagine, that in formula $(10) u_{A}\left(x_{i l}^{e t}\right) \approx u_{A}\left(x_{i l}\right) \approx 1$, correlation coefficient $r$ in one case is equal to minus 1 (pairs of readings are changed in antiphase, if some 
of them increase then the other simultaneously decrease), in the second one it is equal to 0 (readings of the both instruments change randomly) and in the third one it is equal to 1 (pairs of readings change in phase, if they increase and decrease simultaneously). Then experimental standard deviation of single bias is approximately equal to 2 or 1,4 , or 0 .

Thus the below formulae provide the same result. Instead of formulae, (6)-(11) calculate bias for each pair of readings:

$$
d_{i l}=x_{i l}-x_{i l}^{e t} .
$$

If comparison of two measures is provided on the comparator (comparison of two gage blocks or two weights, etc.), then bias $d_{i l}$ between two measures is measured directly and is used for calculation of the quantity value, assigned to the measure. Deviation from nominal value can be assigned to the measure. It means that formulae (12)-(16) can be used for evaluation of standard deviation of comparison.

Average bias in measurement points is calculated by the formula:

$$
d_{i}=\frac{\sum_{l=1}^{m} d_{i l}}{m} .
$$

Experimental standard deviation of single bias in particular measurement point:

$$
u_{A}\left(d_{i l}\right)=S\left(x_{i l}^{e t}, x_{i l}\right)=\sqrt{\frac{\sum_{l=1}^{m}\left(d_{i l}-d_{i}\right)^{2}}{m-1}} .
$$

Experimental standard deviation of average bias in particular measurement point is calculated by the formula:

$$
\begin{aligned}
u_{A}\left(d_{i}\right) & =S\left(\bar{x}_{i}^{e t}, \bar{x}_{i}\right)=\sqrt{\frac{\sum_{l=1}^{m}\left(d_{i l}-d_{i}\right)^{2}}{m \cdot(m-1)}}= \\
& =\frac{u_{A}\left(d_{i l}\right)}{\sqrt{m}}=\frac{S\left(x_{i l}^{e t}, x_{i l}\right)}{\sqrt{m}} .
\end{aligned}
$$

Uncertainty budget should also include the summary standard uncertainty of bias in the measurement point, taken from the measurement standard calibration certificate (the same as corrections to measurement standard readings) or measures, which are calculated by the formula:

$$
u_{c}\left(d_{i}^{e t}\right)=\frac{U\left(d_{i}^{e t}\right)}{k} \text { or } u_{c}\left(x_{i}^{e t}\right)=\frac{U\left(x_{i}^{e t}\right)}{k},
$$

where

$U\left(d_{i}^{e t}\right)$ is expanded uncertainty of bias in the measurement point, taken from the measurement standard calibration certificate;

$U\left(x_{i}^{e t}\right)$ is expanded uncertainty of the measure.

It should be mentioned, that in single measurement mode in the process of calibration it is impossible to obtain standard uncertainties $u_{A}\left(x_{i l}\right)$ or $u_{A}\left(x_{i l}^{e t}\right)$ of single readings of the measuring instrument and measurement standard. Their values can be specified by manual or they should be obtained from previous comparisons or calibrations, for example by model (5). Standard uncertainties of the single readings should be specified by calibration certificate and/or calibration instruction.

\section{Measurement uncertainty connected with resolu-} tion of the indicating measuring instrument and measurement standard

Resolution of the indicating measuring instrument is sometimes called discreteness of measuring instrument readings. That is the last digit of the measuring instrument reading to which the measuring instrument or measurement executor rounds the measurement result. Rounding error can be important component of the measurement uncertainty.

Readings can be rather stable and the same in particular measurement point for some measurement standards and measuring instruments, but it does not mean that measurement uncertainty is equal to zero or the nearest to zero value F.2.2.1 [1]. The last digits of readings can be the same in particular measurement points of the measurement rage or within the measurement range.

Misconception that this component should be always added to the measurement uncertainty exists. This is necessary only if readings of the measurement standard or measuring instrument or comparator are the same notwithstanding the quantity of measurements. If readings of the measuring instrument has changed at least by one unit of the smallest significant digit, then measurement uncertainty component connected with resolution of the indicating measuring instrument will be included to standard deviation of the single reading calculated by the formula (7).

If readings of the measurement standard or measuring instrument or comparator are the same, then according to F.2.2.1 [1] and 3.3.2 [2] standard deviation of the single reading of the measuring instrument and measurement standard or standard deviation of the single bias, instead of formulae (7) and (14) correspondingly is calculated by the formulae:

$$
u_{A}\left(x_{i l}\right)=\frac{\Delta_{a}}{2 \sqrt{3}} ; u_{A}\left(x_{i l}^{e t}\right)=\frac{\Delta_{a}^{e t}}{2 \sqrt{3}} ; u_{A}\left(d_{i l}\right)=\frac{\Delta_{d}}{2 \sqrt{3}} .
$$


$\Delta_{a}, \Delta_{a}^{e t}$ and $\Delta_{d}$ is resolution of the indicating measuring instrument, measurement standard or comparator.

Quantity value measured by the measuring instrument is with equal probability within the interval from $-\Delta_{a} / 2$ to $\Delta_{a} / 2$, therefore formulae (17) are formulated exactly according to F.2.2.1 [1] or formula (3.8) [2].

Let us explain it with the simple example. Assume that 11 series of measurements with $m=10$ measurements in each were obtained within the instrument measurement range. Measuring instrument with the indicating measuring instrument resolution 1 (one unit) was used. Let us present these measurement series in Table 1 omitting all other digits in the measurement process except the last one. Average of the readings and standard deviation of the single reading is also calculated in the Table 1 . The measurement uncertainty component is also included, where it is necessary due to the indicating measuring instrument resolution and standard deviation of readings average is calculated by the formula (8).

Formal standard deviation of single reading of the measuring instrument is equal to zero in the first and the last measurement results series. Existing random measurement error component did not appear. According to the first formula (17) standard deviation of the single reading $S\left(x_{i l}\right)=0,288$ units is obtained for the first and the last series.

Based on the above the conclusion can be that the indicating measuring instrument resolution is the source of several variants of measurement uncertainty calculation. That is, if all readings in the series are the same, then standard measurement uncertainty of the indicating measuring instrument is calculated by the formulae (17), and if at least one or several of the readings differ, then it is calculated by standard formulae (7). If to summary standard measurement uncertainty always add measurement uncertainty component calculated by the formulae (17), then it unreasonably changes uncertainty estimate from the best to the bigger one.

Calculation of the standard deviation of the instruments readings using data from the Table 1 according to the formula (8) is appropriate as random measurement error component fully appears. If equal last digits were obtained for each of 11 series according to Table 1, then use of the formula (8) was not appropriate. Measurement error component due to the indicating measuring instrument resolution should be equal to 0,288 for all series for any quantity of measurements.

\section{Uncertainty by elementary measurement models in} the process of measurement standards and measuring instruments calibration

For easy ordering of measurement uncertainty evaluation cases let us denote calibrated measuring instrument by symbol $I$ (Instrument), and instrument, which is measurement standard by symbol $I^{e t}$ (Instrument - measurement standard (etalon)). If measuring instrument after calibration becomes measurement standard for calibration of the other measurement standards or instruments, then before calibration it is denoted by symbol I (Instrument). Let us denote calibrated measure by symbol $M$ (Measure), and measure, which is measurement standard by symbol $\mathrm{M}^{e t}$ (Measure - measurement standard (etalon)).

Table 1. The last digits of the simulated (assumed) measurement series

\begin{tabular}{|c|c|c|c|c|c|c|c|c|c|c|c|c|c|}
\hline$x_{i 1}$ & $x_{i 2}$ & $x_{i 3}$ & $x_{i 4}$ & $x_{i 5}$ & $x_{i 6}$ & $x_{i 7}$ & $x_{i 8}$ & $x_{i 9}$ & $x_{i I 0}$ & $\bar{x}_{i}$ & $S\left(x_{i l}\right)$ & $S\left(x_{i l}\right)=\frac{\Delta_{a}}{2 \sqrt{3}}$ & $S\left(\bar{x}_{i}\right)$ \\
\hline 0 & 0 & 0 & 0 & 0 & 0 & 0 & 0 & 0 & 0 & 0 & - & 0,288 & 0,091 \\
\hline 1 & 0 & 0 & 0 & 0 & 0 & 0 & 0 & 0 & 0 & 0,1 & 0,316 & - & 0,100 \\
\hline 1 & 1 & 0 & 0 & 0 & 0 & 0 & 0 & 0 & 0 & 0,2 & 0,422 & - & 0,133 \\
\hline 1 & 1 & 1 & 0 & 0 & 0 & 0 & 0 & 0 & 0 & 0,3 & 0,483 & - & 0,153 \\
\hline 1 & 1 & 1 & 1 & 0 & 0 & 0 & 0 & 0 & 0 & 0,4 & 0,516 & - & 0,163 \\
\hline 1 & 1 & 1 & 1 & 1 & 0 & 0 & 0 & 0 & 0 & 0,5 & 0,527 & - & 0,167 \\
\hline 1 & 1 & 1 & 1 & 1 & 1 & 0 & 0 & 0 & 0 & 0,6 & 0,516 & - & 0,163 \\
\hline 1 & 1 & 1 & 1 & 1 & 1 & 1 & 0 & 0 & 0 & 0,7 & 0,483 & - & 0,153 \\
\hline 1 & 1 & 1 & 1 & 1 & 1 & 1 & 1 & 0 & 0 & 0,8 & 0,422 & - & 0,133 \\
\hline 1 & 1 & 1 & 1 & 1 & 1 & 1 & 1 & 1 & 0 & 0,9 & 0,316 & - & 0,100 \\
\hline 1 & 1 & 1 & 1 & 1 & 1 & 1 & 1 & 1 & 1 & 1 & - & 0,288 & 0,091 \\
\hline
\end{tabular}


Based on the above calibration process denoted by the symbols is expressed by the following cases:

- comparing of two measures - measurement standard and calibrated measure $\left(M: M^{e t}\right)$;

- comparing of the measuring instrument readings with value assigned to the measure $\left(I: M^{e t}\right)$;

- measuring by measurement standard of the quantity value reproduced by the calibrated measure, this quantity value will be assigned to the measure as a result of calibration $\left(M: I^{e t}\right)$;

- comparing the readings of two measuring instruments, one of the instruments is measurement standard $\left(I: I^{e t}\right)$.

Two dots between the symbols denote calibration process.

In Tables 2-5, formulae for calculation of summary standard uncertainty components for the various measurement models or measurement cases are put in brackets.

Table 2 is compiled for measurement case $I: I^{e t}$, when readings of two measuring instruments were taken non-synchronously (correlation does not appear), but is not expanded to measures comparing. Table is also compiled for measurement cases $M: I^{e t}$ and $I: M^{e t}$, when the measure is calibrated by the measurement standard (indicating measuring instrument) or the measurement standard (indicating measuring instrument) is calibrated by the measure. Recall, that in terms of this article for clearance the indicating measuring instrument and the measure are not considered as measurement standards before calibration and they become the measurement standards after calibration. Indicating measuring instrument or measure can become the working measuring instrument and the measures after calibration.

Measurement uncertainty components from columns 7 and/or 8 from Table 2 are added to summary standard measurement uncertainty, if corresponding measurement uncertainty components from columns $3,4,5$ and 6 are equal to zero.

Table 3 is compiled for measurement results processing in the process of calibration by formulae (6)-(11) for case $I: I^{e t}$, when readings of two measuring instruments were taken synchronously, that is correlation between the readings appears. Table can be used for case, when measurements by measuring instruments are taken non-synchronously.

Measurement uncertainty components from columns 9 and 10 from Table 3 are added to summary standard measurement uncertainty, if corresponding measurement uncertainty components from columns 3-7 from Table 3 are equal to zero.

Table 4 is compiled for processing the same synchronous or non-synchronous measurement results in the process of calibration by formulae (12)-(16).

Table 2. Summary standard measurement uncertainty components, when measurement standard and calibrated instrument readings are taken non-synchronously

\begin{tabular}{|c|c|c|c|c|c|c|c|c|}
\hline $\begin{array}{c}\text { Measurement } \\
\text { model }\end{array}$ & Case & $u_{A}\left(x_{i l}\right)$ & $u_{A}\left(x_{i l}^{e t}\right)$ & $u_{A}\left(\bar{x}_{i}\right)$ & $u_{A}\left(\bar{x}_{i}^{e t}\right)$ & $\frac{\left(\Delta_{a}\right)}{2 \sqrt{3}}$ & $\frac{\left(\Delta_{a}^{e t}\right)}{2 \sqrt{3}}$ & $\begin{array}{c}u_{c}\left(d_{i}^{e t}\right) \text { or } \\
u_{c}\left(x_{i}^{e t}\right)\end{array}$ \\
\hline \multirow{2}{*}{$d_{i}=x_{i l}-x_{i l}^{e t}$} & 2 & 3 & 4 & 5 & 6 & 7 & 8 & 9 \\
\cline { 2 - 9 } & $M: I^{e t}$ & - & $(7.2)$ & - & - & - & $(17.2)$ & $(16.1)$ \\
\hline \multirow{4}{*}{$d_{i}=\bar{x}_{i}-x_{i I}^{e t}$} & $I: M^{e t}$ & $(7.1)$ & - & - & - & $(17.1)$ & - & $(16.2)$ \\
\cline { 2 - 9 } & $M: I^{e t}$ & - & $(7.2)$ & - & - & - & $(17.2)$ & $(16.1)$ \\
\cline { 2 - 9 } & $I: M^{e t}$ & - & - & $(8.1)$ & - & $(17.1)$ & - & $(16.2)$ \\
\hline \multirow{3}{*}{$d_{i}=x_{i l}-\bar{x}_{i}^{e t}$} & $M: I^{e t}$ & - & - & - & $(8.2)$ & - & $(17.2)$ & $(16.1)$ \\
\cline { 2 - 9 } & $I: M^{e t}$ & $(7.1)$ & - & - & - & $(17.1)$ & - & $(16.2)$ \\
\cline { 2 - 9 } & $I: I^{e t}$ & $(7.1)$ & - & - & $(8.2)$ & $(17.1)$ & $(17.2)$ & $(16.1)$ \\
\hline
\end{tabular}

Table 3. Summary standard measurement uncertainty components, when measurement standard (indicating measuring instrument) and calibrated instrument readings are taken synchronously or non-synchronously

\begin{tabular}{|c|c|c|c|c|c|c|c|c|}
\hline $\begin{array}{c}\text { Measurement } \\
\text { model }\end{array}$ & Case & $u_{A}\left(x_{i l}\right)$ & $u_{A}\left(x_{i l}^{e t}\right)$ & $u_{A}\left(\bar{x}_{i}\right)$ & $u_{A}\left(\bar{x}_{i}^{e t}\right)$ & $\frac{\left(\Delta_{a}\right)}{2 \sqrt{3}}$ & $\frac{\left(\Delta_{a}^{e t}\right)}{2 \sqrt{3}}$ & $u_{c}\left(d_{i}^{e t}\right)$ \\
\hline 1 & 2 & 3 & 4 & 5 & 6 & 7 & 8 & 9 \\
\hline$d_{i}=x_{i l}-x_{i l}^{e t}$ & $I: I^{e t}$ & $(7.1)$ & $(7.2)$ & - & - & $(17.1)$ & $(17.2)$ & $(16.1)$ \\
\hline$d_{i}=\bar{x}_{i}-\bar{x}_{i}^{e t}$ & $I: I^{e t}$ & - & - & $(8.1)$ & $(8.2)$ & $(17.1)$ & $(17.2)$ & $(16.1)$ \\
\hline
\end{tabular}


Table 4. Summary standard measurement uncertainty components, when measurement standard (indicating measuring instrument) and calibrated instrument readings are taken synchronously or non-synchronously and processed by formulae (12)-(16)

\begin{tabular}{|c|c|c|c|c|c|c|}
\hline $\begin{array}{c}\text { Measurement } \\
\text { model }\end{array}$ & Case & $u_{A}\left(d_{i l}\right)$ & $u_{A}\left(d_{i}\right)$ & $\frac{\left(\Delta_{a}\right)}{2 \sqrt{3}}$ & $\frac{\left(\Delta_{a}^{e t}\right)}{2 \sqrt{3}}$ & $u_{c}\left(d_{i}^{e t}\right)$ \\
\hline 1 & 2 & 3 & 4 & 5 & 6 & 7 \\
\hline$d_{i}=x_{i l}-x_{i l}^{e t}$ & $I: I^{e t}$ & $(14)$ & - & $(17.1)$ & $(17.2)$ & $(16.1)$ \\
\hline$d_{i}=\bar{x}_{i}-\bar{x}_{i}^{e t}$ & $I: I^{e t}$ & - & $(15)$ & $(17.1)$ & $(17.2)$ & $(16.1)$ \\
\hline
\end{tabular}

Table 5. Summary standard measurement uncertainty components for case comparing of two measures

\begin{tabular}{|c|c|c|c|c|c|}
\hline $\begin{array}{c}\text { Measurement } \\
\text { model }\end{array}$ & Case & $u_{A}\left(d_{i l}\right)$ & $u_{A}\left(d_{i}\right)$ & $\frac{\left(\Delta_{d}\right)}{2 \sqrt{3}}$ & $u_{c}\left(x_{i}^{e t}\right)$ \\
\hline 1 & 2 & 3 & 4 & 5 & 6 \\
\hline$d_{i}=x_{i l}-x_{i}^{e t}$ & $M: M^{e t}$ & $(14)$ & - & $(17.3)$ & $(16.2)$ \\
\hline$d_{i}=\bar{x}_{i}-x_{i}^{e t}$ & $M: M^{e t}$ & - & $(15)$ & $(17.3)$ & $(16.2)$ \\
\hline
\end{tabular}

Measurement uncertainty components from columns 5 and 6 from Table 4 are added to summary standard measurement uncertainty, if corresponding measurement uncertainty components from columns 3 and 4 from Table 4 are equal to zero.

Summary standard measurement uncertainty evaluation for case $M: M^{e t}$ comparing of two measures should be provided taking into account, that measurand is $d_{i}$, quantity value $x_{i}^{e t}$ is known from previous calibrations and defined quantity value is calculated by the formula $x_{i}=x_{i}^{e t}-d_{i}$.

Measurement uncertainty components from columns 5 from Table 5 are added to summary standard measurement uncertainty, if corresponding measurement uncertainty components from columns 3 and 4 from Table 5 are equal to zero.

Let us demonstrate several examples of Table 1-5 use for summary standard measurement uncertainty calculation.

For measurement model $d_{i}=x_{i 1}-x_{i 1}^{e t}$ (2) in case $I: M^{e t}$ (Table 2):

$$
u_{c}\left(y_{i}\right)=u_{c}\left(d_{i}\right)=\sqrt{\frac{\left(\Delta_{a}\right)^{2}}{12}+u_{c}\left(x_{i}^{e t}\right)^{2}+\ldots}
$$

if $u_{A}\left(x_{i 1}\right)=0$;

$$
u_{c}\left(y_{i}\right)=u_{c}\left(d_{i}\right)=\sqrt{u_{A}\left(x_{i 1}\right)^{2}+u_{c}\left(x_{i}^{e t}\right)^{2}+\ldots}
$$

if $u_{A}\left(x_{i l}\right) \neq 0$.

For measurement model $d_{i}=\bar{x}_{i}-x_{i l}^{e t}$ (3) in case $I: M^{e t}$ (Table 2):

$$
u_{c}\left(y_{i}\right)=u_{c}\left(d_{i}\right)=\sqrt{\frac{\left(\Delta_{a}\right)^{2}}{12 \cdot m}+u_{c}\left(x_{i}^{e t}\right)^{2}+\ldots}
$$

if $u_{A}\left(\bar{x}_{i}\right)=0$;

$$
u_{c}\left(y_{i}\right)=u_{c}\left(d_{i}\right)=\sqrt{u_{A}\left(\bar{x}_{i}\right)^{2}+u_{c}\left(x_{i}^{e t}\right)^{2}+\ldots}
$$

if $u_{A}\left(\bar{x}_{i}\right) \neq 0$.

For measurement model $d_{i}=x_{i l}-\bar{x}_{i}^{e t}(4)$ in case $I: I^{e t}$ (Table 2):

$$
\begin{gathered}
u_{c}\left(y_{i}\right)=u_{c}\left(d_{i}\right)= \\
=\sqrt{u_{A}\left(\bar{x}_{i}^{e t}\right)^{2}+\frac{\left(\Delta_{a}\right)^{2}}{12}+u_{c}\left(d_{i}^{e t}\right)^{2}+\ldots}
\end{gathered}
$$

if $u_{A}\left(x_{i 1}\right)=0 ; u_{A}\left(\bar{x}_{i}^{e t}\right) \neq 0$;

$$
\begin{gathered}
u_{c}\left(y_{i}\right)=u_{c}\left(d_{i}\right)= \\
=\sqrt{u_{A}\left(x_{i 1}\right)^{2}+\frac{\left(\Delta_{a}^{e t}\right)^{2}}{12 \cdot m}+u_{c}\left(d_{i}^{e t}\right)^{2}+\ldots}
\end{gathered}
$$

if $u_{A}\left(x_{i 1}\right) \neq 0 ; u_{A}\left(\bar{x}_{i}^{e t}\right)=0$.

For measurement model $d_{i}=\bar{x}_{i}-\bar{x}_{i}^{e t}(5)$ in case $I: I^{e t}$ and using formulae (6)-(11), (Table 3):

$$
u_{c}\left(y_{i}\right)=u_{c}\left(d_{i}\right)=
$$


Table 6. Calibration results

\begin{tabular}{|c|c|c|c|c|c|}
\hline $\begin{array}{c}\text { Point } \\
\text { number }\end{array}$ & $\begin{array}{c}\text { Average } \\
\text { value }\end{array}$ & $\begin{array}{c}\text { Quantity value } \\
\text { of measurement } \\
\text { standard }\end{array}$ & Bias & $\begin{array}{c}\text { Expanded } \\
\text { uncertainty } \\
\text { of bias k=2 }\end{array}$ & $\begin{array}{c}\text { Standard deviation } \\
\text { of the single reading }\end{array}$ \\
\hline$i$ & $\bar{x}_{i}$ & $x_{i}{ }^{e t}$ & $d_{i}$ & $U\left(d_{i}\right)$ & $u_{A}\left(x_{i l}\right)=S\left(x_{i l}\right)$ \\
\hline
\end{tabular}

Table 7. Calibration results

\begin{tabular}{|c|c|c|c|c|c|}
\hline $\begin{array}{c}\text { Point } \\
\text { number }\end{array}$ & $\begin{array}{c}\text { Quantity value } \\
\text { of measurement } \\
\text { standard }\end{array}$ & $\begin{array}{c}\text { Average } \\
\text { value }\end{array}$ & Correction & $\begin{array}{c}\text { Expanded } \\
\text { uncertainty of } \\
\text { correction k=2 }\end{array}$ & $\begin{array}{c}\text { Standard deviation } \\
\text { of the single reading }\end{array}$ \\
\hline$i$ & $x_{i}^{e t}$ & $\bar{x}_{i}$ & $\Delta x_{i}$ & $U\left(\Delta x_{i}\right)$ & $u_{A}\left(x_{i l}\right)=S\left(x_{i l}\right)$ \\
\hline
\end{tabular}

$$
=\sqrt{\frac{\left(\Delta_{a}\right)^{2}}{12 \cdot m}+\frac{\left(\Delta_{a}^{e t}\right)^{2}}{12 \cdot m}+u_{c}\left(d_{i}^{e t}\right)^{2}+\ldots}
$$

if $u_{A}\left(\bar{x}_{i}\right)=0, u_{A}\left(\bar{x}_{i}^{e t}\right)=0$ та $u_{A}\left(d_{i}\right)=0$.

For measurement model $d_{i}=\bar{x}_{i}-\bar{x}_{i}^{e t}(5)$ in case $I: I^{e t}$ and using formulae (12)-(16), (Table 4):

$$
u_{c}\left(y_{i}\right)=u_{c}\left(d_{i}\right)=\sqrt{u_{A}\left(d_{i}\right)^{2}+u_{c}\left(d_{i}^{e t}\right)^{2}+\ldots}
$$

if $u_{A}\left(d_{i}\right) \neq 0$.

Expanded uncertainty, which is put to calibration certificate, is calculated by the formula:

$$
U\left(d_{i}\right)=k \cdot u_{c}\left(d_{i}\right)
$$

where

$k$ is expansion coefficient by certain confidence probability.

For example for measurement model $d_{i}=\bar{x}_{i}-x_{i l}^{e t}$, which is put to calibration certificate, calibration results are presented in the tabled form (Table 6 or Table 7).

Calibration results demonstrated in Tables 6 and 7 can be used for calibration of the other working measurement standards or working measuring instruments. Thus, measurement results processing of the repeated calibrations is provided by the formulae (1)(26). This exact calibration chain provides measurement traceability. The last column in Tables 6 and 7 is used if measurement standard is used for measurement standards of lower rank calibration including the measures and the working measuring instruments in single measurement mode.

Data from Tables 6 and 7 can be used for calculation of operational measurement uncertainty by measuring and experimental laboratories of the enterprise.

Biases in intermediate calibration points, that is in calibration points of the measuring instrument, are calculated by interpolation measurement model:

$$
y_{t}=-d_{i i+1}^{t} \text { or } y_{t}=\Delta x_{i i+1}^{t},
$$

Bias in that case is interpolated by the formula:

$$
d_{i i+1}^{t}=d_{i}+\left(d_{i+1}-d_{i}\right) \cdot \frac{x_{i i+1}^{t}-x_{i}}{x_{i+1}-x_{i}} .
$$

where

$d_{i i+1}^{t}$ is bias of the measuring instrument readings $x_{i i+1}^{t}$ in the point with number $t$, which is between points with numbers $i$ and $i+1$ of instruments' calibration points;

$d_{i}$ and $d_{i+1}$ are biases of the measuring instrument readings $x_{i}$ and $x_{i+1}$ in points with numbers $i$ and $i+1$ of instruments' calibration points;

$x_{i i+1}^{t}$ are measuring instrument readings in point with number $t$.

Measurement uncertainty of bias is also calculated by interpolation method:

$$
\begin{gathered}
u\left(\Delta x_{i i+1}^{t}\right)=u\left(d_{i i+1}^{t}\right)= \\
=u\left(d_{i}\right)+\left(u\left(d_{i+1}\right)-u\left(d_{i}\right)\right) \cdot \frac{x_{i i+1}^{t}-x_{i}}{x_{i+1}-x_{i}}
\end{gathered}
$$

\section{Example of elementary measurement models use in the calibration process}

Let us consider measurement results processing in the measuring instrument calibration process by elementary measurement model (3):

$$
d_{i}=\bar{x}_{i}-x_{i 1}^{e t} .
$$

Processed simulated measuring instruments readings are put in Table 8 . That is, readings are randomly generated numbers and are not related to any real measuring instrument.

Prior processing of the calibration results is provided in Table 8. Measuring instrument calibration results are calculated in it, they are equal for all measurement models, particularly average readings values 
Table 8. Simulated measuring instrument readings and their prior processing results

\begin{tabular}{|c|c|c|c|c|c|c|c|c|c|c|c|c|c|}
\hline \multirow{2}{*}{$i$} & \multicolumn{10}{|c|}{ Measuring instrument readings } & \multirow{2}{*}{$\bar{x}_{i}$} & \multirow{2}{*}{$x_{i}^{e t}$} & \multirow{2}{*}{$d_{i}$} \\
\hline & $x_{i 1}$ & $x_{i 2}$ & $x_{i 3}$ & $x_{i 4}$ & $x_{i 5}$ & $x_{i 6}$ & $x_{i 7}$ & $x_{i 8}$ & $x_{i 9}$ & $x_{i 10}$ & & & \\
\hline 1 & 0,5 & 0,5 & 0,5 & 0,5 & 0,5 & 0,5 & 0,5 & 0,5 & 0,5 & 0,5 & 0,50 & 0,60 & $-0,10$ \\
\hline 2 & 0,9 & 0,9 & 0,9 & 0,9 & 0,9 & 0,9 & 0,9 & 0,9 & 0,9 & 0,9 & 0,90 & 1,00 & $-0,10$ \\
\hline 3 & 1,4 & 1,4 & 1,4 & 1,4 & 1,5 & 1,4 & 1,4 & 1,4 & 1,4 & 1,4 & 1,41 & 1,45 & $-0,04$ \\
\hline 4 & 2,0 & 1,9 & 2,0 & 2,0 & 1,9 & 2,1 & 2,1 & 2,0 & 2,0 & 2,0 & 2,00 & 2,00 & 0,00 \\
\hline 5 & 2,5 & 2,5 & 2,4 & 2,5 & 2,4 & 2,5 & 2,5 & 2,5 & 2,5 & 2,5 & 2,48 & 2,40 & 0,08 \\
\hline 6 & 3,6 & 3,7 & 3,7 & 3,7 & 3,6 & 3,6 & 3,6 & 3,6 & 3,6 & 3,7 & 3,64 & 3,50 & 0,14 \\
\hline 7 & 4,9 & 4,7 & 4,7 & 4,7 & 4,7 & 4,8 & 4,8 & 4,8 & 4,7 & 4,8 & 4,76 & 4,50 & 0,26 \\
\hline 8 & 5,9 & 5,7 & 5,9 & 5,9 & 5,9 & 6,0 & 5,8 & 5,8 & 5,9 & 6,0 & 5,88 & 5,60 & 0,28 \\
\hline 9 & 7,9 & 7,9 & 8,0 & 8,0 & 8,1 & 7,9 & 7,8 & 8,0 & 8,0 & 7,8 & 7,94 & 7,60 & 0,34 \\
\hline 10 & 11,1 & 11,2 & 11,0 & 11,0 & 11,1 & 11,2 & 11,2 & 11,0 & 11,0 & 11,2 & 11,10 & 10,70 & 0,40 \\
\hline
\end{tabular}

Table 9. Measurement uncertainty evaluation by measurement model (3)

\begin{tabular}{|c|c|c|c|c|c|c|c|c|c|}
\hline & \multicolumn{2}{|c|}{$\begin{array}{c}\text { Average of measuring } \\
\text { instrument readings, } \\
\text { measurement standard } \\
\text { readings and bias }\end{array}$} & \multicolumn{2}{|c|}{$\begin{array}{c}\text { Experimental } \\
\text { standard deviation } \\
\text { of single reading }\end{array}$} & \multicolumn{2}{|c|}{ Uncertainty budget } & \multicolumn{2}{|c|}{$\begin{array}{c}\text { Summary and expanded } \\
\text { uncertainty }\end{array}$} \\
\cline { 2 - 11 }$i$ & $\bar{x}_{i}$ & $x_{i}{ }^{e t}$ & $d_{i}$ & $S\left(x_{i l}\right)$ & $S\left(x_{i l}\right)=\frac{\Delta_{a}}{\sqrt{12}}$ & $u_{A}\left(\bar{x}_{i}\right)=\frac{S\left(x_{i l}\right)}{\sqrt{m}}$ & $u_{c}\left(x_{i}{ }^{e t}\right)$ & $u_{c}\left(d_{i}\right)$ & $U\left(y_{i}\right)=U\left(d_{i}\right)$ \\
\hline 1 & 0,50 & 0,60 & $-0,10$ & - & 0,029 & 0,009 & 0,010 & 0,013 & 0,026 \\
\hline 2 & 0,90 & 1,00 & $-0,10$ & - & 0,029 & 0,009 & 0,010 & 0,013 & 0,026 \\
\hline 3 & 1,41 & 1,45 & $-0,04$ & 0,032 & - & 0,010 & 0,010 & 0,014 & 0,028 \\
\hline 4 & 2,00 & 2,00 & 0,00 & 0,067 & - & 0,021 & 0,010 & 0,023 & 0,046 \\
\hline 5 & 2,48 & 2,40 & 0,08 & 0,042 & - & 0,013 & 0,010 & 0,016 & 0,032 \\
\hline 6 & 3,64 & 3,50 & 0,14 & 0,052 & - & 0,016 & 0,010 & 0,019 & 0,038 \\
\hline 7 & 4,76 & 4,50 & 0,26 & 0,070 & - & 0,022 & 0,010 & 0,024 & 0,048 \\
\hline 8 & 5,88 & 5,60 & 0,28 & 0,092 & - & 0,029 & 0,010 & 0,031 & 0,062 \\
\hline 9 & 7,94 & 7,60 & 0,34 & 0,097 & - & 0,031 & 0,010 & 0,033 & 0,066 \\
\hline 10 & 11,10 & 10,70 & 0,40 & 0,094 & - & 0,030 & 0,010 & 0,032 & 0,064 \\
\hline
\end{tabular}

of the measuring instrument $\bar{x}_{i}$ in the calibration process and measurement biases $d_{i}$ in all calibration points.

Measurement uncertainty of bias by elementary measurement model (3) is provided in Table 9. For the first and the second rows, calculation is provided according to the formula (20) and for the rest of rows according to the formula (21).

\section{Conclusions}

1. Elementary measurement models can be used for calibration methods development for wide range of working measurement standards and working measuring instruments.
2. If calibrated measurement standard is used for the single direct measurements in the process of repeated calibration and measurement, then according to users request experimental standard deviation of the single reading together with biases and their uncertainties can be determined in the points of instrument calibration.

3 . Taking the same readings of measuring instrument in measurement point, its standard deviation of the single reading can be calculated depending on resolution of the indicating measuring instrument.

4. Correct choice of the measurement uncertainty components by various models and cases allows obtaining the best (not overestimated or underestimated) uncertainty evaluation. 
Посилання

1. JCGM 100:2008, Evaluation of Measurement Data - Guide to the Expression of Uncertainty in Measurement, Joint Committee for Guides in Metrology (JCGM), France, 2008. www.BIPM.org.
2. EA-4/02 M:2013 Evaluation of the Uncertainty of Measurement in Calibration, 2013.

www.european-accreditatin.org 